\title{
Encoding and controlling of two droplet trains in a microfluidic network with the loop-like structure
}

\author{
Kui Song ${ }^{1}$ Guoqing $\mathrm{Hu}^{2} \cdot$ Xiaoling $\mathrm{Hu}^{1} \cdot$ Runtao Zhong $^{3,4} \cdot$ Xiaohui Wang $^{3}$ • \\ Bingcheng Lin ${ }^{3,4}$
}

Received: 13 May 2015 / Accepted: 11 September 2015 / Published online: 28 September 2015

(C) Springer-Verlag Berlin Heidelberg 2015

\begin{abstract}
A theoretical model is derived mathematically for the encoding and controlling of the navigating of two droplet trains in a microfluidic network with a loop-like structure. The model reveals the relationship between the new outlet droplet train's arrangement information (output signals) and the parameters including the two droplet trains' input signals (droplet intervals), tuning flow rates, etc. The theoretical results are compared with the experimental results and they agree with each other. We find that every tuning flow rate corresponds to a certain output signal and a new droplet train can be obtained accurately. The generation orders of the successive droplets of the new droplet train remain unchanged within a certain range of the tuning flow rates. This work can be a useful reference for traffic controlling of two or more droplet trains in many microfluidic networks including the loop structure; the output signal of this work can be the input one for the next level which makes the multilevel studies possible. In
\end{abstract}

Electronic supplementary material The online version of this article (doi:10.1007/s10404-015-1651-8) contains supplementary material, which is available to authorized users.

Kui Song

songkui-25@163.com

1 College of Civil Engineering and Mechanics, Xiangtan University, Xiangtan 411105, China

2 The State Key Laboratory of Nonlinear Mechanics, Institute of Mechanics, Chinese Academy of Sciences, Beijing 100190, China

3 Department of Biotechnology, Dalian Institute of Chemical Physics, Chinese Academy of Sciences, Dalian 116000, China

4 Research Center of Lab on a Chip (RCLOC), Dalian 116000, China addition, this study can help to promote the effective fusion of droplets and further the biological and chemical applications on droplet microfluidics.

Keywords Droplet trains · Encoding · Loop-like structure $\cdot$ Droplet intervals $\cdot$ Fusion

\section{Introduction}

Droplet microfluidics has many applications in chemistry, biology, medical science and material synthesis (Song et al. 2003; Brouzes et al. 2009; Huebner et al. 2007; Takinoue and Takeuchi 2011; Mazutis et al. 2009; Bogojevic et al. 2012; Miller et al. 2012; Liu et al. 2012). One interesting application based on microfluidic droplets or bubbles is to conduct logic functions operations and information encoding and decoding (Prakash and Gershenfeld 2007; Fuerstman et al. 2007). Prakash and Gershenfeld (2007) proposed bubble logic operations of AND/OR/NOT gates, a toggle flip-flop, a ring oscillator and timing restoration in special microfluidic devices. Fuerstman et al. (2007) realized the operations of encrypting and decrypting signals within a microfluidic encoding/decoding loop device, by treating the droplet intervals as the coded signals. In those microchannel networks, the presence of junctions, bypasses and loops notably increase the complexity of the fluid dynamics of droplets, sometimes resulting in multiperiodicity and multistability (Jousse et al. 2006; Belloul et al. 2009; Jeanneret et al. 2012; Wu et al. 2012; Gleichmann et al. 2014).

One of the key challenges in droplet applications is how to effectively control droplets motion after their generation in microfluidic devices. Much work has been done for generation, splitting, sorting, fusion and traffic control of droplets (Thorsen et al. 2001; Anna et al. 2003; Murran and 
Najjaran 2012; Cristobal et al. 2006; Link et al. 2006; Zhou and Yao 2013; Barbier et al. 2006; Fu et al. 2014; Gleichmann et al. 2014). Theoretical models are also proposed to explain and predict the dynamic behaviors of droplets, and thus provide better controlling of droplet traffic. In theoretical analysis, the relationship of pressure $\Delta P$, flow rate $Q$ and flow resistance $R, \Delta P=R Q$, is similar to the Ohm's law in electric circuit and often used to simulate, optimize and predict the droplet motion in microchannels (Ajdari 2004; Schindler and Ajdari 2008; Song et al. 2012; Maddala et al. 2013; Zanella and Biral 2014). Jousse et al. (2006) derived a compact model of multiphase liquid-liquid flow in fluidic networks by using the electrical analogy between laminar flow and electric current. Schindler and Ajdari (2008) proposed a simplified model to find robust dynamical behavior of droplets and quantify its response to the changes in flow conditions and geometrical parameters of the microchannels. Belloul et al. (2009) studied the competition mechanism between local collision for small droplets and collective hydrodynamic feedback for large ones. Wu et al. (2014) studied bubble coalescence at the T-junction. They observed colliding and squeezing coalescence and investigated the coalescence efficiency by changing the capillary number, bubble size, liquid viscosity and so on.

In addition, a droplet may either split into smaller daughter droplets at large capillary numbers (Song et al. 2003; Link et al. 2004) or choose to enter the channel that has the instantaneous maximum flow rate (Sessoms et al. 2009; Engl et al. 2005) when reaching a junction in microchannels. Salkin et al. (2013) found that the breakup of droplets is affected by the additional presence of droplets in downstream channels and the slug-to-slug interactions, so it is with the breakup of bubbles at a microfluidic T-junction (Fu et al. 2014), and the flow resistance due to droplets or bubbles should be considered for the design of microfluidic devices. The essentially nonconstant flow resistance of each droplet is determined by the viscosity ratio of the two fluids, droplet volume, velocity and other parameters. Although many studies assume a constant one and can obtain the essential characteristics of the droplet dynamics as an approximation for analysis (Schindler and Ajdari 2008; Cybulski and Garstecki 2010; Bithi and Vanapalli 2010; Garstecki 2010), droplets motion will still result in nonlinear change of the flow resistance of microchannels and affect the flow rates of the channels and later choices of droplets at the junctions in turn. Actually, this problem is a complex nonlinear feedback regulation one (Sessoms et al. 2009; Engl et al. 2005), which brings great difficulties to the setup of theoretical models even for the droplet dynamics in the simple microfluidic devices (Willaime et al. 2006; Garstecki et al. 2005), and more research work including the usage of innovative analytical methods should be done.
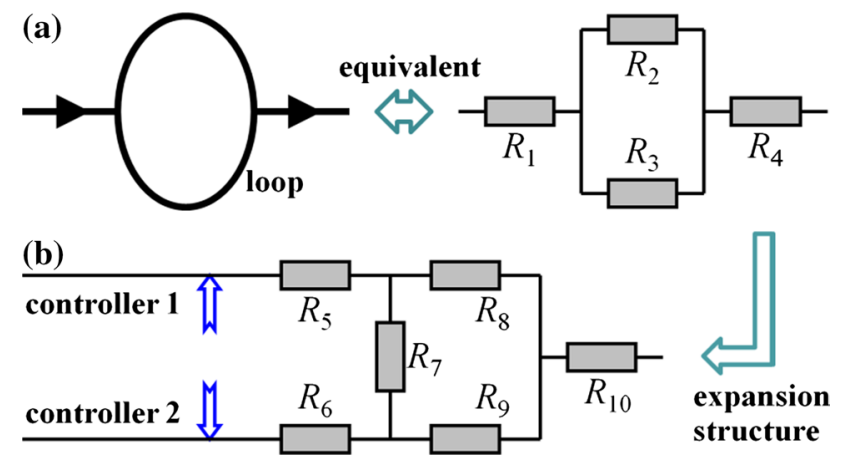

Fig. 1 a Schematic of the loop structure and its equivalent electrical circuit. b The expansion circuit structure which can achieve two droplet trains' input and operation. $R_{1}-R_{10}$ are the corresponding flow resistances

Otherwise, the relative time or space intervals of highly monodisperse droplets remain unchanged in the same microchannel and thus can form encoding signals in the channel-based microflow systems (Behzad et al. 2010; Fuerstman et al. 2007); but the fusion of the droplets in this condition is difficult. On the contrary, polydispersity of droplets cannot form constant encoding signals because of the difference in droplet velocities, and it may lead to uncontrolled fusion (Mazutis and Griffiths 2009). The fusion is a basic and crucial step for droplets as microreactors, and the fusion efficiency depends on the proximity extent of the droplets (Niu et al. 2009; Ahn et al. 2006; Mostowfi et al. 2007; Wu et al. 2014), so droplets encounter controlling is important.

Previous work about droplet encoding and fusion is independent; here we concern highly monodisperse droplets in microdevices and combine both encoding and encounter behaviors of droplets together. We believe that a certain interval means encoding signals, while encounter means fusion in theory in the outlet channel, so we take droplets fusion as the special case of their encoding. Another motivation of this research work is that droplet encoding research is often confined to one droplet train passing a loop (Jousse et al. 2006; Fuerstman et al. 2007; Belloul et al. 2009; Labrot et al. 2009; Jeanneret et al. 2012; Wu et al. 2012). Controlling of two droplet trains has more extensive applications. Zheng et al. (2004) used two droplet trains for the first time to form droplet pairs and index the composition of droplets, and they applied this technology for the screening conditions of the protein crystallization. Okushima et al. (2004) used the two droplet trains to produce double emulsions with different inner compartments, which can be applied for the analysis of confined chemical reactions, biological screening, drug delivery systems and so on. 
Fig. 2 Schematic of the microfluidic network. The network contains two T-junctions which generate two droplet trains, two dilution or concentration modules, a loop-like structure and one outlet channel. After generation, droplets undergo four stages $I, I I, I I I$ and $I V$; finally, they leave the network from the outlet channel with different patterns. Droplets are represented with black hollow and solid circles. Related parameters are marked in the schematic

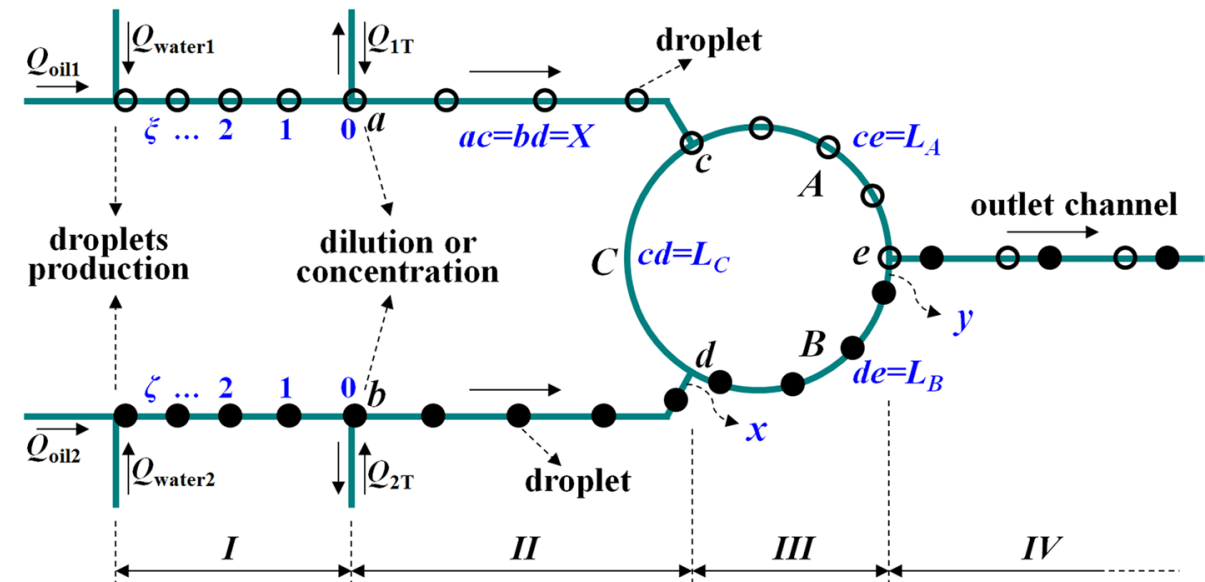

It is believed that a loop device can be looked as an electrical circuit as shown in Fig. 1a. For one input channel correspondingly to one droplet train, we think Fig. 1b can be an expansion structure for encoding research of two droplet trains. Meanwhile, we impose two controllers to add the flexible manipulation; the controllers can be dilution or concentration modules and the imposed controlling flow rates can be called tuning flow rates (Sessoms et al. 2009; Yamada et al. 2008). We do mathematical analysis and establish an effective and robust theoretical solution to study the droplet dynamics correspond to the expansion structure (Fig. 1b), and all of these are to achieve our droplet encoding and fusion research thought. What we want to get is an analytical model which can give the control conditions of the two droplet trains' outlet information and can guide the realizations of droplet logic functions and fusion behaviors. After analysis, we find the model can also control droplets of different generation sequences to reach the specified dynamic locations to form information. A waterin-oil (W/O) droplet microfluidics experiment is also done to test the model as an example.

In this paper, the theory model setup is presented in Sect. 2. The experimental demonstration is given in Sect. 3. Section 4 gives the theoretical results and compares them with experimental results by changing the parameters. The motion state analysis of droplets is also given in this section as a further discussion according to the simple rule that droplets will choose to enter the channel which has the instantaneous maximum flow rate. Section 5 concludes the paper.

\section{Theoretical modeling of droplet traffic}

The basic yet essential structure considered here is a threenode loop with two inlets and one outlet. According to the flow-electric current analogy in laminar flows, such loop can be represented by an electric circuit in Fig. 1b. The electric resistances correspond to the flow resistances of the microchannel segments between nodes, respectively. To further control the motion of the liquid and droplets, additional controlling channels can be added to the upstream parts of the network to form an expanded structure. If the droplets present in the microfluidic network, the behavior of the flow becomes more complex than the case of singlephase flow. Thus, it is necessary to develop efficient models to describe and predict the droplet traffic, especially for the large complex microfluidic networks. In this section, we derive the mathematical relationship between the outlet patterns (the intervals of droplets) and the parameters including microstructure size, tuning flow rates and so on. With mathematical analysis, theory model can be looked as a useful numerical simulation method to understand and manage the microfluidic droplet-related traffic problems (Schindler and Ajdari 2008; Cybulski and Garstecki 2010; Jousse et al. 2006; Sessoms et al. 2009; Behzad et al. 2010; Sessoms et al. 2010; Smith and Gaver 2010; Glawder et al. 2011). The analytical results here can provide effective reference for encoding and encounter of droplets.

The expansion circuit structure in Fig. $1 \mathrm{~b}$ can be explained with a further detailed schematic as shown in Fig. 2, with relevant parameters describing the operating of the droplet motion through the loop structure. The loop shown in the network is selected for convenience, and it may be other forms which can be called the loop-like structures. The constant cross section of microchannel network is symmetrical and contains two identical microfluidic T-junctions that generate two droplet trains at the upstream. The total flow rates are $Q_{1}$ (including the continuous fluid flow rate $Q_{\text {oill }}$ and the dispersed fluid flow rate $Q_{\text {water1 }}$ ) for the upper T-junction for droplet generation and $Q_{2}$ (including the continuous fluid flow rate $Q_{\text {oil2 }}$ and the dispersed fluid flow rate $Q_{\text {water2 }}$ ) for the lower T-junction. These two T-junctions then produce two trains of droplets which are denoted by $\xi$ for the upper train and $\zeta$ for the lower train. Two tuning flows of $Q_{1 T}$ and $Q_{2 T}$ are introduced at 
nodes $a$ and $b$, respectively. We let $Q_{1}=n_{1} Q, Q_{2}=n_{2} Q$, $Q_{1 T}=m_{1} Q$ and $Q_{2 T}=m_{2} Q$, where $n_{1}, n_{2}, m_{1}$ and $m_{2}$ are nondimensional factors. The droplet intervals then will be increased or decreased (dilution or concentration), depending on the direction of the tuning flow. The train of droplets moves through the nodes $a$ (or $b$ ), $c$ (or $d$ ) and then $e$, which can be categorized by four stages: I, II, III and IV, as shown in Fig. 2. Finally, a new droplet train will be re-grouped in the outlet channel. Therefore, the primary goal of this work is to establish the relationship between the rearranged droplets and the incoming droplets. The arrangement information of the new droplet train is related to the operating conditions and geometrical parameters:

$\lambda^{\mathrm{IV}}=F\left(Q, n_{1}, n_{2}, m_{1}, m_{2}, X, L_{A}, L_{B}, \ldots\right)$

where $\lambda^{\mathrm{IV}}$ represents droplet intervals of the output droplet train in stage IV, and $X, L_{A}$ and $L_{B}$ are the lengths of the channel segments betweens nodes as shown in Fig. 2.

In general, when number $\xi$ droplet of train 1 arrives at node $c$, number $\zeta$ droplet of train 2 will arrive at the location that has a distance of $x$ away from node $d$. Due to the symmetrical nature of the network structure, $x$ satisfies the condition: $0 \leq x<\lambda_{2}^{\text {II }}$. Here the subscript denotes droplet train 1 or 2, while the superscript denotes the different stages I, II, III and IV. For example, $\lambda_{2}^{\text {II }}$ represents the adjacent droplets' interval of droplet train 2 at stage II. The total time for droplet $\xi$ of train 1 to arrive at node $c$ is $\Delta t_{1, \xi}=\Delta t_{1}^{\mathrm{II}}+\xi \lambda_{1}^{\mathrm{I}} / V_{1}^{\mathrm{I}}(\xi \geq 0)$, where $\Delta t_{1}^{\mathrm{II}}=X / V_{1}^{\mathrm{II}}, V_{1}^{\mathrm{I}}$ and $V_{1}^{\mathrm{II}}$ are the droplet velocities, and the subscript represents droplet train 1 and the superscript represents the stages I and II that droplets stay in; similarly, the total time for droplet $\zeta$ of train 2 to arrive at the location that has an $x$ distance away from node $d$ is $\Delta t_{2, \zeta}=\Delta t_{2}^{\mathrm{II}}+\zeta \lambda_{2}^{\mathrm{I}} / V_{2}^{\mathrm{I}}(\zeta \geq 0)$, where $\Delta t_{2}^{\mathrm{II}}=(X-x) / V_{2}^{\mathrm{II}}$. $V_{2}^{\mathrm{I}}$ and $V_{2}^{\mathrm{II}}$ are the corresponding droplet velocities. Considering $\Delta t_{1, \xi}=\Delta t_{2, \zeta}$, we obtain:

$X / V_{1}^{\mathrm{II}}+\xi \lambda_{1}^{\mathrm{I}} / V_{1}^{\mathrm{I}}=(X-x) / V_{2}^{\mathrm{II}}+\zeta \lambda_{2}^{\mathrm{I}} / V_{2}^{\mathrm{I}}$

Droplet velocities at the four stages can be calculated as:

$$
\left\{\begin{array}{l}
V_{1}^{\mathrm{I}}=\beta Q_{1} / S ; \\
V_{2}^{\mathrm{I}}=\beta Q_{2} / S ; \\
V_{1}^{\mathrm{II}}=\beta\left(Q_{1}+Q_{1 T}\right) / S ; \\
V_{2}^{\mathrm{II}}=\beta\left(Q_{2}+Q_{2 T}\right) / S ; \\
V_{1}^{\mathrm{III}}=\beta Q_{A} / S ; \\
V_{2}^{\mathrm{III}}=\beta Q_{B} / S ; \\
V^{\mathrm{IV}}=\beta Q_{\text {out }} / S .
\end{array}\right.
$$

where $S$ is the cross-sectional area of the microchannel, $Q_{A}$ and $Q_{B}$ are the corresponding flow rates in the segmental channels $A$ and $B$, respectively, and $Q_{\text {out }}$ is the flow rate in the outlet channel. The slip factors of droplet velocity for all the channels are assumed to be constant (Labrot et al.

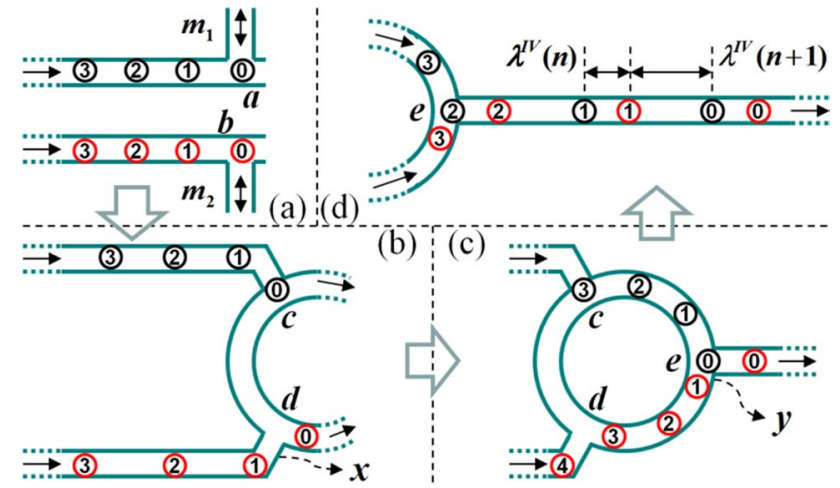

Fig. 3 A diagram of droplets traffic and encoding process for the case of $\xi=0, \zeta=1, i=j=0$. a-d are four important states of the traffic process in turn. Droplets, represented with black and red circles, are labeled and their sequence numbers are written in them for convenient description. Droplet number 0 (black) arrives at node $c$, while droplet number 1 (red) arrives at the location that has a distance of $x$ away from node $d$; meanwhile, droplet number 0 (black) becomes the one $i=0$, droplet number 1 (red) becomes the one $j=0$. From the next state at node $e$, we get $i=j=0$. A new droplet train is formed in the outlet channel at last (color figure online)

2009; Bithi and Vanapalli 2010; Jeanneret et al. 2012) and marked with $\beta$ in Eq. (3).

Substituting Eq. (3) into Eq. (2) yields the controlling equation of $x$ :

$\frac{\xi \lambda_{1}^{\mathrm{I}}}{Q_{1}}+\frac{X}{Q_{1}+Q_{1 T}}=\frac{\zeta \lambda_{2}^{\mathrm{I}}}{Q_{2}}+\frac{X-x}{Q_{2}+Q_{2 T}}$

Equation (4) can be changed into:

$\frac{x}{X}=1-\frac{m_{2}+n_{2}}{m_{1}+n_{1}}-\frac{\xi\left(m_{2}+n_{2}\right)}{n_{1}} \frac{\lambda_{1}^{\mathrm{I}}}{X}+\frac{\zeta\left(m_{2}+n_{2}\right)}{n_{2}} \frac{\lambda_{2}^{\mathrm{I}}}{X}$

where $0 \leq x / X<\left(m_{2}+n_{2}\right) \lambda_{2}^{I} /\left(n_{2} X\right)$. Figure $3 \mathrm{a}$, $\mathrm{b}$ shows droplets traffic and encoding processes for two incoming droplet trains in the case of $\xi=0$ and $\zeta=1$ (Fig. 3a-d is four times the steady motion state). As the flow rates $Q_{1}, Q_{2}$ and segment length $X$ are fixed, the distance of $x$ is solely determined by two tuning flow rates $\left(m_{1} Q\right.$ and $\left.m_{2} Q\right)$.

Under proper values of tuning flow rates, droplets from either part do not go through segment channel $C$ and the whole flow system reaches a steady state. We introduce $i$ to indicate an arbitrary droplet following droplet $\xi$ of train 1 and $j$ to indicate an arbitrary droplet following droplet $\zeta$ of train 2 . Here $i \geq 0, j \geq 0$, and $i=0$ is droplet $\xi$ of train 1 while $j=0$ is droplet $\zeta$ of train 2 . Generally, we can still assume when droplet $i$ arrives at node $e$, droplet $j$ will arrive at the location that has a distance of $y$ away from node $e ; y$ satisfies the condition: $0 \leq y<\lambda_{2}^{\text {III }}$. Because each droplet has a length, when $y$ becomes smaller than this length, collisions will occur at the outlet junction, and there will be another limitation for $y$. In this paper, we make assumptions that the length of droplets is not 
considered for convenience of the theoretical modeling, and thus the model is more valid for short droplets. Figure $3 \mathrm{a}-\mathrm{c}$ shows droplets traffic and encoding processes for two incoming droplet trains in the case of $\xi=0, \zeta=1$ and $i=j=0$. Finally, we can rearrange the droplet train in the outlet microchannel by varying the tuning flow rates as shown in Fig. 3d.

Following the same procedure described before, the total time for number $\xi+i$ droplet of train 1 to arrive at node $e$ is $\Delta t_{1, \xi+i}=\Delta t_{1}^{\mathrm{III}}+i \lambda_{1}^{\mathrm{II}} / V_{1}^{\mathrm{II}}(i \geq 0)$, where $\Delta t_{1}^{\mathrm{III}}=L_{A} / V_{1}^{\mathrm{IIII}}$; the total time for number $\zeta+j$ droplet of train 2 to arrive at the location that has a $y$ distance away from node $e$ is $\Delta t_{2, \zeta+j}=\Delta t_{2}^{\mathrm{III}}+\left(x+j \lambda_{2}^{\mathrm{II}}\right) / V_{2}^{\mathrm{II}}(j \geq 0)$ where $\Delta t_{2}^{\text {III }}=\left(L_{B}-y\right) / V_{2}^{\text {III }}$. Depending on the capillary number, droplet will deform at nodes and take time when passing through the nodes. Here we make assumptions that the droplets are short and ignore the droplet deformation and relaxation time. It is more convenient for theoretical modeling, and the model is more valid for short droplets mentioned above. Hence, based on the approximate equation $\Delta t_{1, \xi+i} \approx \Delta t_{2, \zeta+j}$, we obtain:

$L_{A} / V_{1}^{\mathrm{III}}+i \lambda_{1}^{\mathrm{II}} / V_{1}^{\mathrm{II}}=\left(L_{B}-y\right) / V_{2}^{\mathrm{III}}+\left(x+j \lambda_{2}^{\mathrm{II}}\right) / V_{2}^{\mathrm{II}}$

Substituting Eq. (3) into Eq. (6) yields the controlling equation of $y$ :

$\frac{i \lambda_{1}^{\mathrm{II}}}{Q_{1}+Q_{1 T}}+\frac{L_{A}}{Q_{A}}=\frac{x+j \lambda_{2}^{\mathrm{II}}}{Q_{2}+Q_{2 T}}+\frac{L_{B}-y}{Q_{B}}$

The droplet intervals at all stages can be derived as follows (see Appendix 1 for details):

$$
\left\{\begin{array}{l}
\lambda_{1}^{\mathrm{II}}=\left(Q_{1}+Q_{1 T}\right) \lambda_{1}^{\mathrm{I}} / Q_{1} ; \\
\lambda_{2}^{\mathrm{II}}=\left(Q_{2}+Q_{2 T}\right) \lambda_{2}^{\mathrm{I}} / Q_{2} ; \\
\lambda_{1}^{\mathrm{III}}=Q_{A} \lambda_{1}^{\mathrm{II}} /\left(Q_{1}+Q_{1 T}\right) ; \\
\lambda_{2}^{\mathrm{III}}=Q_{B} \lambda_{2}^{\mathrm{II}} /\left(Q_{2}+Q_{2 T}\right) ; \\
\lambda_{1}^{\mathrm{IV}}=Q_{\text {out }} \lambda_{1}^{\mathrm{III}} / Q_{A} ; \\
\lambda_{2}^{\mathrm{IV}}=Q_{\text {out }} \lambda_{2}^{\mathrm{III}} / Q_{B} .
\end{array}\right.
$$

Using the relationship in Eq. (7), Eq. (8) can be changed to:

$\frac{i \lambda_{1}^{\mathrm{I}}}{Q_{1}}+\frac{L_{A}}{Q_{A}}=\frac{x+j\left(Q_{2}+Q_{2 T}\right) \lambda_{2}^{\mathrm{I}} / Q_{2}}{Q_{2}+Q_{2 T}}+\frac{L_{B}-y}{Q_{B}}$

For Stokes flows, microfluidic network can be analyzed based on its equivalent Ohmic circuit. In the loop structure shown in Fig. 2, we derive the flow rates in segments $A$, $B$ and $C$ by assuming that the flow in segment $C$ moves counterclockwise:

$\left\{\begin{array}{l}Q_{C}=\frac{R_{A}\left(Q_{1}+Q_{1 T}\right)-R_{B}\left(Q_{2}+Q_{2 T}\right)}{R_{A}+R_{B}+R_{C}} \\ Q_{A}=\left(Q_{1}+Q_{1 T}\right)-Q_{C} \\ Q_{B}=\left(Q_{2}+Q_{2 T}\right)+Q_{C} .\end{array}\right.$ where $R_{A}, R_{B}$ and $R_{C}$ are the total hydrodynamic resistances of segments $A, B$ and $C$, respectively. With the presence of droplets, the total hydrodynamic resistances are the channel resistances plus the resistances caused by droplets, i.e., $R_{A}=\overline{R_{A}}+n_{A} R_{d 1}, R_{B}=\overline{R_{B}}+n_{B} R_{d 2}$ and $R_{C}=\overline{R_{C}}$ (there are no droplets in segment $C$ ), where $\overline{R_{A}}, \overline{R_{B}}$ and $\overline{R_{C}}$ are the pure resistances of the segments, $R_{d 1}$ and $R_{d 2}$ are the resistances caused by one droplet from the upper and lower train, respectively. $n_{A}=L_{A} / \lambda_{1}^{\mathrm{III}}$ and $n_{B}=L_{B} / \lambda_{2}^{\mathrm{III}}$ are the numbers of droplets in segment $A$ and segment $B$, respectively. $R_{d 1}$ and $R_{d 2}$ depend on the parameters such as droplet viscosity $\left(\mu_{d 1}\right.$ and $\left.\mu_{d 2}\right)$, flow rate $\left(Q_{A}\right.$ and $\left.Q_{B}\right)$, viscosity ratio of droplet to continuous phase, surface tension, droplet size (Bretherton 1961; Park and Homsy 1984; Wong et al. 1995). Together with the tuning flow rates, we can change these parameters to control droplet encoding results.

We define $R=\overline{R_{C}}$ and rewrite other resistances in term of $R: \overline{R_{A}}=m_{3} R, \overline{R_{B}}=m_{4} R, R_{d 1}=\Gamma_{1} R$ and $R_{d 2}=\Gamma_{2} R$, where $m_{3}, m_{4}, \Gamma_{1}$ and $\Gamma_{2}$ are dimensionless parameters. Different $\Gamma_{1}$ and $\Gamma_{2}$ stand for two different droplet trains. Combining with the previous definitions, Eq. (9) can be modified to:

$$
\begin{aligned}
\frac{y}{X} & =F \frac{x}{X}+F\left(m_{2}+n_{2}\right)\left(\frac{j \lambda_{2}^{\mathrm{I}}}{n_{2} X}-\frac{i \lambda_{1}^{\mathrm{I}}}{n_{1} X}\right) \\
& +\left(m_{4}-\frac{F m_{3}}{1+\frac{m_{1}+n_{1}}{m_{2}+n_{2}}-F}\right) \frac{L_{C}}{X}
\end{aligned}
$$

where $F$ is:

$F=1+\frac{\left(m_{3}+\Gamma_{1} n_{A}\right) \frac{m_{1}+n_{1}}{m_{2}+n_{2}}-\left(m_{4}+\Gamma_{2} n_{B}\right)}{1+m_{3}+m_{4}+\Gamma_{1} n_{A}+\Gamma_{2} n_{B}}$

According to the Eq. (8), we can derive:

$\left\{\begin{array}{l}\lambda_{1}^{\mathrm{IV}}=Q_{\text {out }} \lambda_{1}^{\mathrm{I}} / Q_{1} \\ \lambda_{2}^{\mathrm{IV}}=Q_{\text {out }} \lambda_{2}^{\mathrm{I}} / Q_{2} .\end{array}\right.$

Since $Q_{\text {out }}=Q_{1}+Q_{2}+Q_{1 T}+Q_{2 T}=\left(m_{1}+m_{2}+n_{1}+n_{2}\right) Q$, we have:

$\left\{\begin{array}{l}\lambda_{1}^{\mathrm{IV}}=\left(m_{1}+m_{2}+n_{1}+n_{2}\right) \lambda_{1}^{\mathrm{I}} / n_{1} \\ \lambda_{2}^{\mathrm{IV}}=\left(m_{1}+m_{2}+n_{1}+n_{2}\right) \lambda_{1}^{\mathrm{II}} / n_{2} .\end{array}\right.$

Equation (14) implies that once two trains of droplets enter the outlet channel, the intervals of droplets change to new values that are determined by tuning flows and droplet interval in the upstream. These droplets from segment $A$ and segment $B$ are combined to form a new train of droplets. Generally, we can get the following coding arrangement:

$\lambda^{\mathrm{IV}}(n)= \begin{cases}V^{\mathrm{IV}} y / V_{2}^{\mathrm{III}}, \quad(n=1) ; \\ \lambda_{1}^{\mathrm{IV}}-\lambda^{\mathrm{IV}}(n-1), \quad(n=3,5,7, \ldots) ; \\ \lambda_{2}^{\mathrm{IV}}-\lambda^{\mathrm{IV}}(n-1), \quad(n=2,4,6, \ldots) .\end{cases}$ 
where $y / V_{2}^{\mathrm{III}} \leq \lambda_{1}^{\mathrm{III}} / V_{1}^{\mathrm{III}}$ and $n$ is the order number of droplets in the new droplet train.

\section{Experimental explanation}

We did a simple experiment to test the model as an example. In experiments, the SU-8 photoresist was used to fabricate molding master on a glass substrate by soft-lithography with mask design shown in Fig. 4a. PDMS prepolymer and curing agent were mixed in the ratio of 10:1 (v/v),
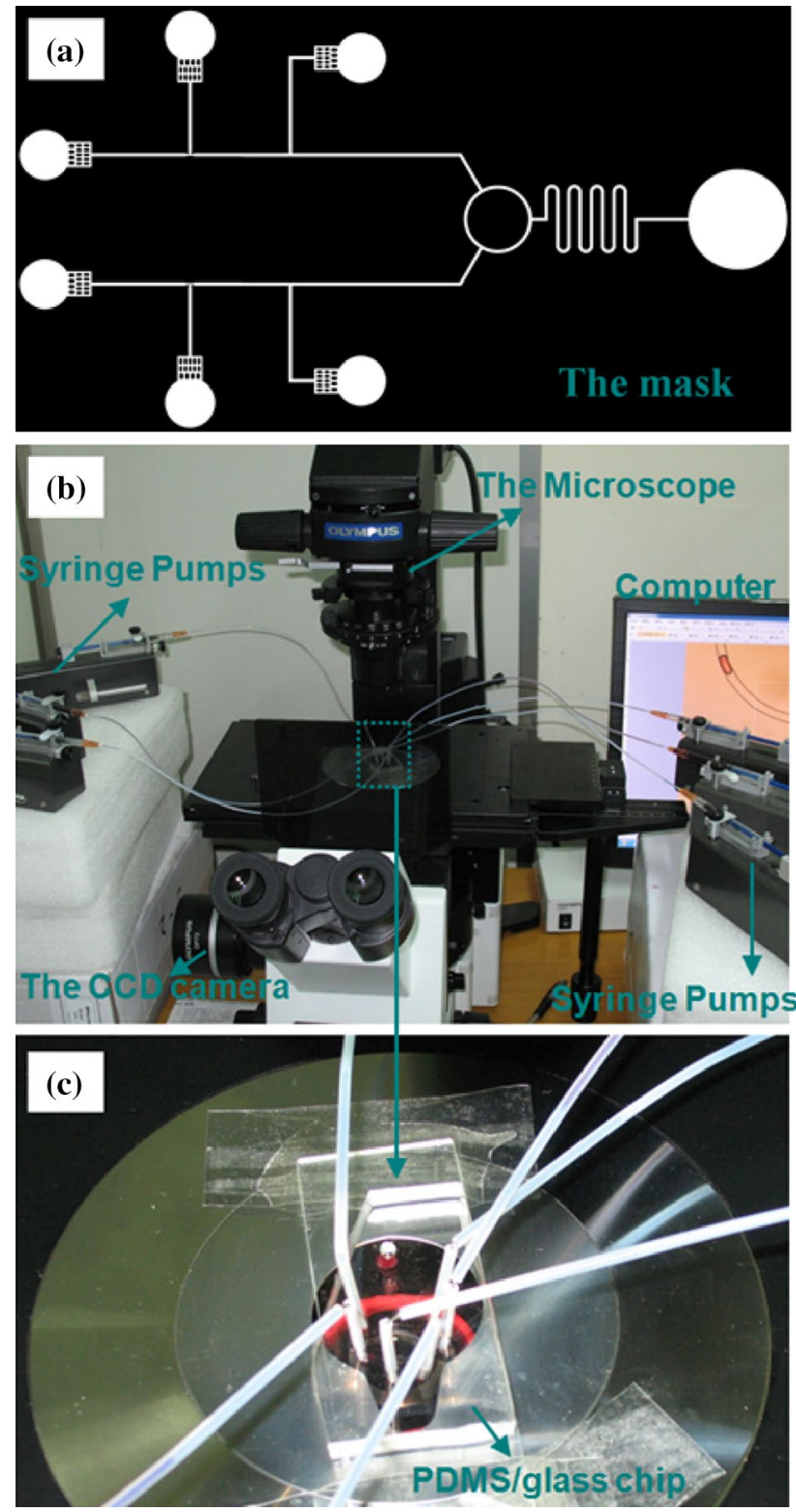

Fig. 4 a Mask of the PDMS/glass chip for soft-lithography. b The actual experimental setup. $\mathbf{c}$ The microfluidic chip and its connection poured on the molding master and degassed. After polymerization at $80^{\circ} \mathrm{C}$ for $2 \mathrm{~h}$, we got a PDMS slab containing desired microstructures by peeling-off and hole-drilling. A glass slide was spin-coated with a thin layer of PDMS and bonded with the PDMS slab after plasma treatment to obtain the hybrid PDMS/glass chip. According to the mask design and Sect. 2, we have $L_{C}=2 \mathrm{~mm}, X=6.56 \mathrm{~mm}$ and $m_{3}=m_{4}=1$; meanwhile, the cross section of the microchannels in the PDMS/glass chip has a height of $60 \mu \mathrm{m}$ and a width of $100 \mu \mathrm{m}$. For droplet generation, the continuous phase was mineral oil with Span-80 surfactant $(1 \%, w / w)$ and the discrete phase was deionized water. The longer pumps (TJ-3A/W0109-1B) were used to inject fluids into the chip. An Olympus microscope and its supporting camera (IX73 inverted microscope; CCD-DP73) were used to observe the droplet motion and take the experimental images (Fig. 4b). The Image-Pro Plus 5.1 and MATLAB softwares were used to treat the images. We controlled the flow rates of the two fluids to generate droplet trains through two T-junctions: $0.6 \mu \mathrm{L} / \mathrm{min}$ for the mineral oil and $0.15 \mu \mathrm{L} / \mathrm{min}$ for the deionized water. The tuning flow rates $Q_{1 T}$ is fixed to $0.3 \mu \mathrm{L} / \mathrm{min}$ and $Q_{2 T}$ is made equal to $0.15,0.2,0.25,0.3,0.35,0.4,0.45,0.5,0.55,0.6,0.65,0.7$, $0.75,0.8$ and $0.85 \mu \mathrm{L} / \mathrm{min}$, respectively. In order to show the experimental results more clearly, we make one droplet train black with Photoshop as Hashimoto and Whitesides (2010), and the results are shown in Figs. 5 and 7.

\section{Results and discussion}

In this section, we show the results with experimental numeric input to the solution. Generally, we mainly show and discuss the results that are controlled by $m_{2}$, keeping other parameters unchanged. We give the controlling results of $x$ and $y$, then the encoding or fusion results, and compare them with the experiment results. Finally we discuss and give the controlling range of $m_{2}$ by droplet motion state analysis.

\subsection{Controlling results of $x$ and $y$}

According to the theoretical analysis, the controlling results of $x$ and $y$ are the basis for getting the controlling results of droplet encoding and fusion in the outlet channel. The nondimensional parameters mentioned in Sect. 2 are used. In the experiment, we get $m_{1}=0.4, \lambda_{1}^{\mathrm{I}}=\lambda_{1}^{\mathrm{II}}=\lambda_{0} \approx 340 \mu \mathrm{m}$ by calculation and measurement under the conditions $n_{1}=n_{2}=1, Q_{1}=Q_{2}=Q=0.75 \mu \mathrm{L} / \mathrm{min}, Q_{1 T}=0.3 \mu \mathrm{L} /$ min and so on (see Sect. 3). Equation (5) becomes:

$$
\frac{x}{X}=(\zeta-\xi)\left(1+m_{2}\right) \frac{\lambda_{0}}{X}+\frac{m_{1}-m_{2}}{1+m_{1}}
$$



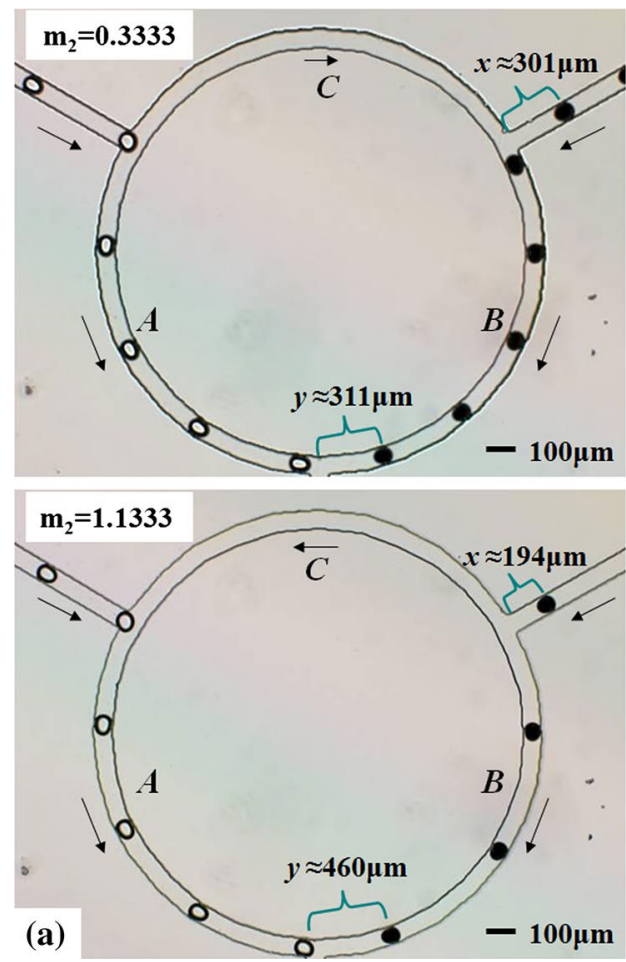

Fig. 5 Results of $x / X$ and $y / X$ controlled by $m_{2}$. a The experimental results of $x$ and $y$ when $m_{2}$ equals to 0.3333 and 1.1333 , respectively. b The controlling results of $x / X$ change with $m_{2}$ and are compared with the experimental results. Here $m_{1}=0.4$ and $k=\zeta-\xi$, different values of $k$ stand for different situations about two droplets of each train arriving at locations of $c$ and $d$ separately and simultaneously. The red dashed line, whose function is $x / X=\left(1+m_{2}\right) \lambda_{0} / X$,

The experimental results of $x$ and $y$ are obtained through measurement as shown in Fig. 5a; in the two cases, $m_{2}$ equals to 0.3333 and 1.1333 , respectively. The partial results of $x / X$ as a function $m_{2}$ according to Eq. (16) are shown in Fig. 5b, where $m_{2}$ ranges from 0.0273 to 1.1971 . The area between the abscissa and the red dashed line is the range of $x / X$; here the function of the red dashed line is $x / X=\left(1+m_{2}\right) \lambda_{0} / X$. In the figure, the artificial marks of the black dashed lines indicate that $x / X$ can be got by a given value of $m_{2}$ under the order of droplets. Here we introduce $k=\zeta-\xi$, where $k=0, \pm 1, \pm 2, \ldots$. Different $k$ represents the different patterns how the droplets of two trains arrive at the two locations. In effect, both the values of $x / X$ and $k$ can be determined by a certain $m_{2}$. For example, when $0.3053<m_{2} \leq 0.4$, we get $k=0$ from Fig. $5 b$; substituting $m_{2}$ and $k$ into Eq. (16), we can get the value of $x / X$.

Based on the controlling results of $x / X$, we let $\Gamma_{1}=\Gamma_{2}=8$ in this paper (Labrot et al. 2009); in the meantime, we derive the average values of the droplet number in branch $A\left(n_{A}\right)$ and branch $B\left(n_{B}\right)$ of the loop by steady-state analysis (see Appendix 2). Finally, we get the results of
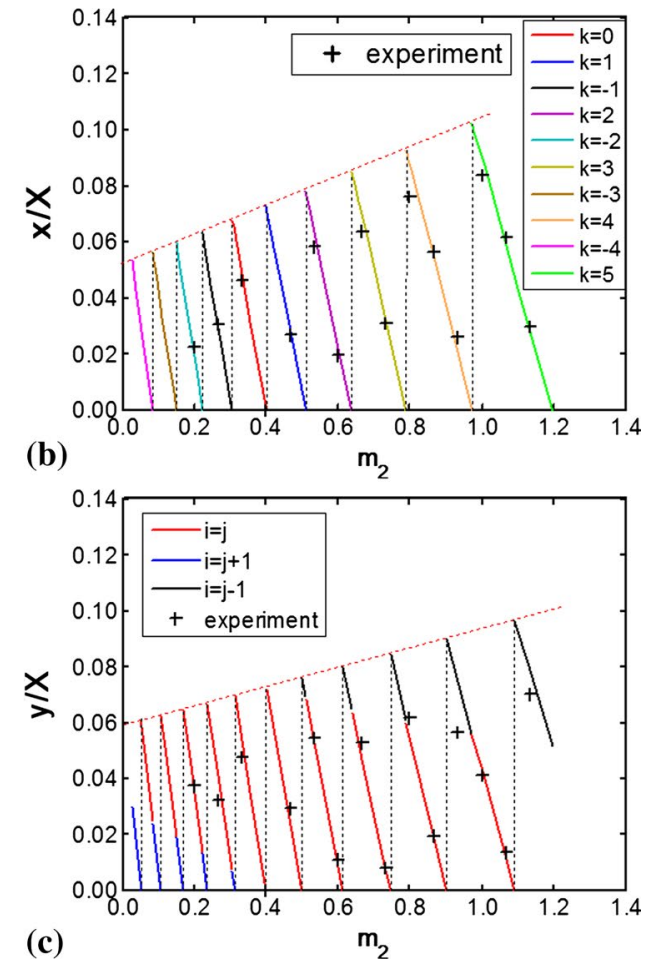

stands for the maximum values of $x / X$ under different values of $k$. Both $x / X$ and $k$ can be determined by $m_{2}$. $\mathbf{c}$ The controlling results of $y / X$ change with $m_{2}$ and are compared with the experimental results. $y / X$ can be determined by $m_{2}$ under the relationship of $i$ and $j$. The red dashed line stands for the maximum values of $y / X$ under droplets order and its function is $y / X=\lambda_{2}^{\text {III }} / X$ (color figure online)

$y / X$ by substituting all the related results into Eq. (11); $y / X$ is a function of $m_{2}$ and the result is shown in Fig. 5c. Similarly, the area between the abscissa and the red dashed line is the range of $y / X$; the function of the red dashed line is $y / X=\lambda_{2}^{I I I} / X$; the artificial marks of the black dashed lines indicate that $y / X$ can be got by a given $m_{2}$ under the order of droplets (expressed by $k$ and the relationship of $i$ and $j$ ). For a certain $m_{2}$, we can know $k$ and the relationship of $i$ and $j$ from Fig. 5b, c and then determine $y / X$ from Eq. (11). Figure $5 \mathrm{c}$ also shows that if the $m_{2}$ is different, the relationship of $i$ and $j$ may also be different under a same $k$. For example, under the condition of $0.9023<m_{2} \leq 1.0909$, $k=1, i$ and $j$ have two relationships: $i=j$ (indicated with the red line) and $i=j-1$ (indicated with the black line). The experimental results are given out with plus symbols in the figures, and they agree with the analytical results very well.

\subsection{Encoding and encounter results of droplets}

Based on the above controlling results of $y / X$ under the experimental conditions, Eq. (15) can be simplified as: 


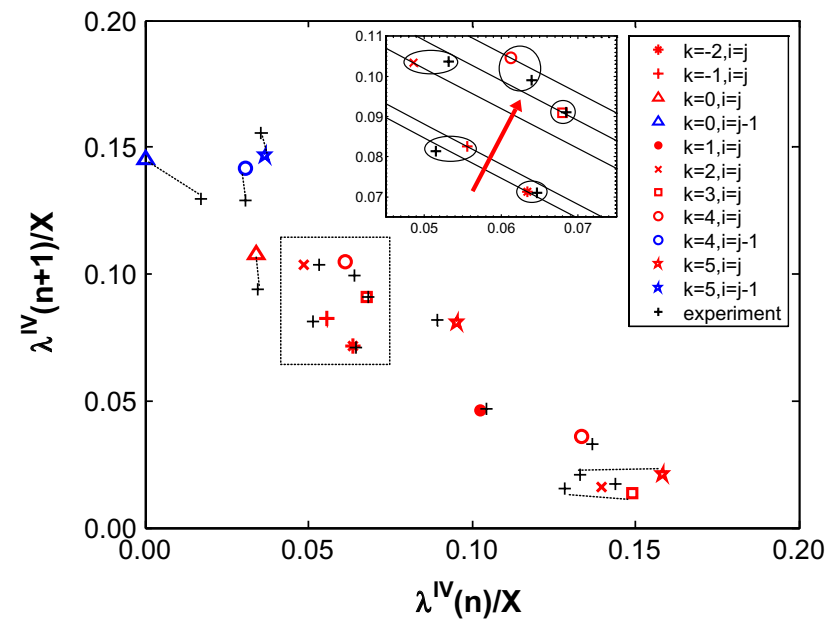

(a)

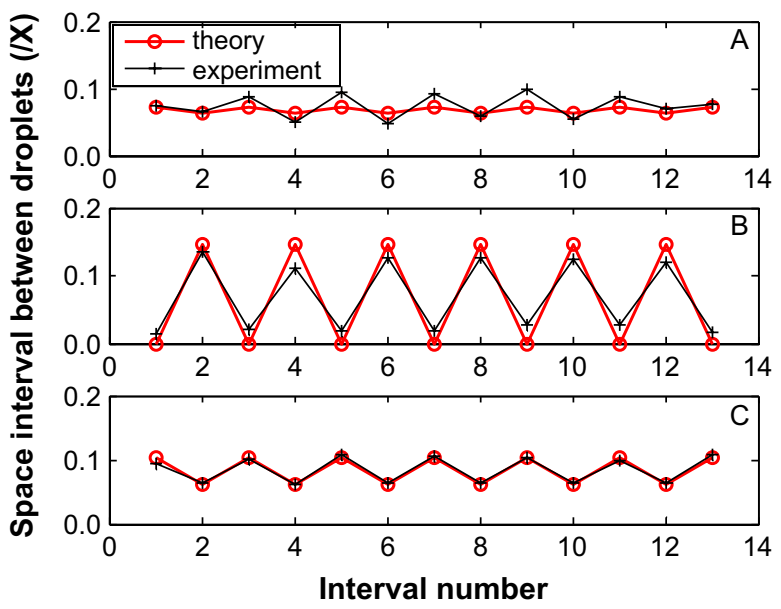

(b)

Fig. 6 Droplets encoding and encounter results under the experimental conditions. a Poincaré maps of the $(n+1)$ st space interval $\left(\lambda^{\mathrm{IV}}(n+1)\right)$ versus the $n$th interval $\left(\lambda^{\mathrm{IV}}(n)\right)$, the value of $m_{2}$ is different for every point. The inset figure describes the sum of $\lambda^{\mathrm{IV}}(n) / X$ and $\lambda^{I V}(n+1) / X$, and the sum increases with $m_{2}$. b Plots from A to $\mathrm{C}$ show signal encoding results of space interval, and their values of $m_{2}$ are A 0.2 , B 0.4 and C 0.8 . Specifically, plot B indicates the encounter state of the two droplet trains

$\left\{\begin{array}{l}\lambda^{\mathrm{IV}}(n+1)=V^{\mathrm{IV}} y / V_{2}^{\mathrm{III}} ; \\ \lambda^{\mathrm{IV}}(n)=\delta-\lambda^{\mathrm{IV}}(n+1) .\end{array}\right.$

where $\delta=\lambda_{1}^{\mathrm{IV}}=\lambda_{2}^{\mathrm{IV}}=\left(2+m_{1}+m_{2}\right) \lambda_{0}, n$ is the order number of droplets in the new droplet train.

Figure 6 shows droplets encoding and encounter results in the outlet channel. Figure $6 \mathrm{a}$ is the Poincaré map that plots the $(n+1)$ st space interval $\left(\lambda^{\mathrm{IV}}(n+1)\right)$ versus the $n$th interval $\left(\lambda^{\mathrm{IV}}(n)\right)$; the intervals are normalized by $X$. The solutions of $\lambda^{\mathrm{IV}}(n)$ and $\lambda^{\mathrm{IV}}(n+1)$ are got from Eq. (17). Each point in the map corresponds to a special value of $m_{2}$. The analytical and experimental results agree

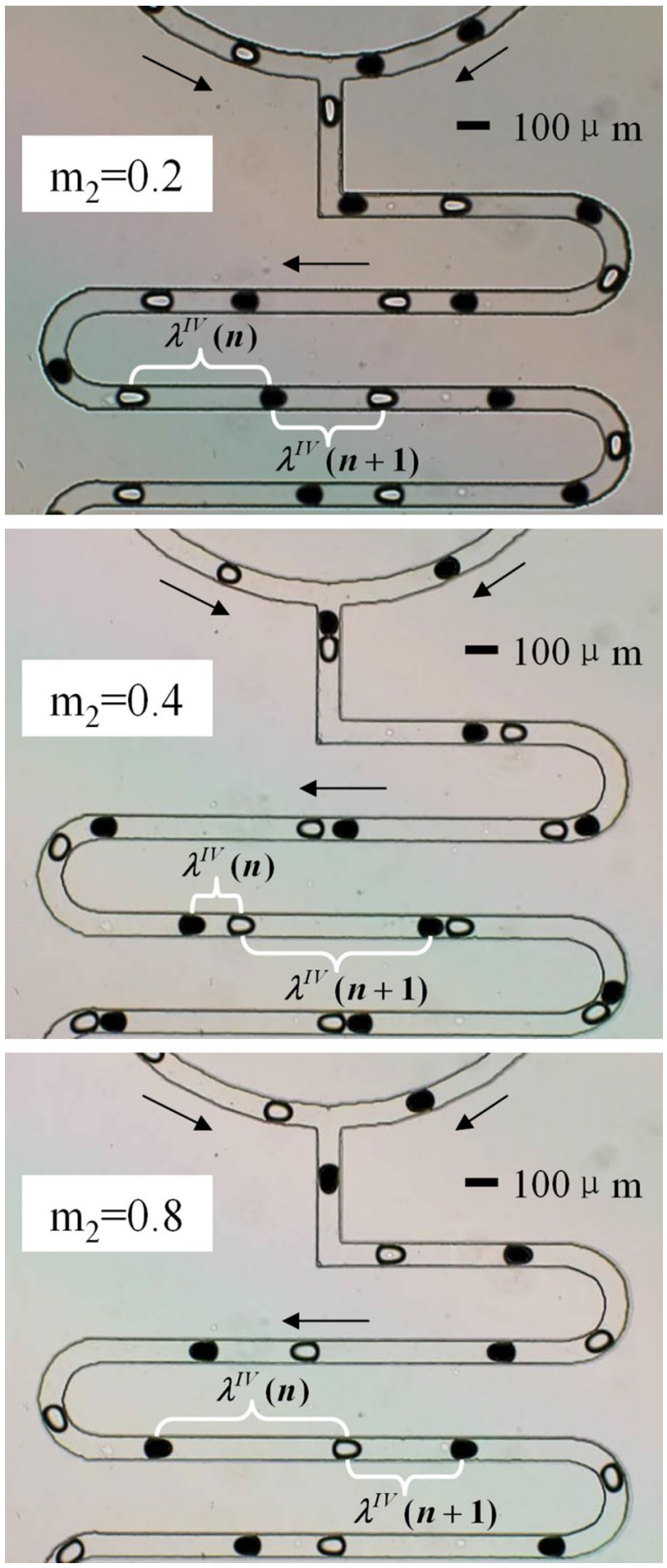

Fig. 7 Experimental results for different $m_{2}$. The space intervals were got by measurement

well with each other, there are several pairs have bigger difference, and we connect them with black dashed lines as shown in Fig. 6a. When $m_{2}$ increases gradually, the sum of the abscissa and the ordinate corresponding 

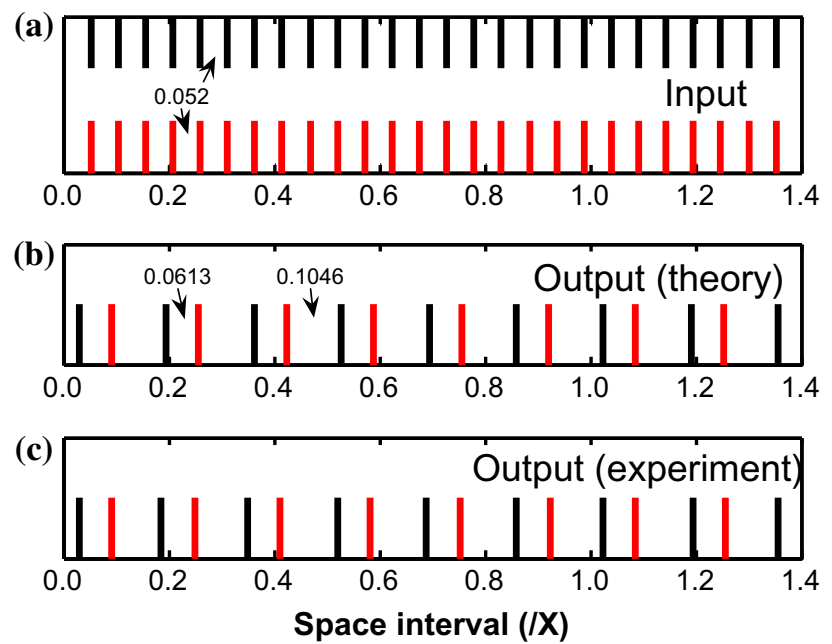

Fig. 8 Schematic for input and output results of droplets space intervals. a represents input, and $\mathbf{b}$ and $\mathbf{c}$ represent theoretical and experimental output results, respectively. Red and black line segments indicate the presence of droplets. Here $m_{2}=0.8$ and $\lambda_{0} / X=0.052$, the new droplet intervals are: $\lambda^{\mathrm{IV}}(n) / X=0.0613$ and $\lambda^{\mathrm{IV}}(n+1) / X=0.1046$ (color figure online)

to every point increases in the linear way; this is because $\lambda^{\mathrm{IV}}(n) / X+\lambda^{\mathrm{IV}}(n+1) / X=\left(2.4+m_{2}\right) / 19.3$ according to the Eq. (14) under the conditions, and it is an increasing function in $(0.0273,1.1971)$. For discrete $m_{2}$, it increases in the leaping way as shown in the inset figure which describes the square area framed by black dashed lines. In the inset figure, the black solid lines is the theory results and the red arrow points to the increasing direction; the theoretical results and their corresponding experimental results are surrounded with ellipses. Figure $6 \mathrm{~b}$ shows the space intervals of droplets versus interval numbers, where $m_{2}$ equals to $0.2,0.4$ and 0.8 from A to $\mathrm{C}$, respectively. The two inlet droplet trains form a new train in the staggered manner at last, and the regular distribution result of the new droplet train can be controlled and taken as the encoding signals. Figure 7 shows the corresponding experimental results, and we measure the distance to get $\lambda^{\mathrm{IV}}(n)$ and $\lambda^{\mathrm{IV}}(n+1)$. In the condition of $m_{2}=0.4, \lambda^{\mathrm{IV}}(n) / X=0$ and $\lambda^{\mathrm{IV}}(n+1) / X=0.1451$, the two droplet trains overlap completely and droplets from the two trains encounter in pairs. We can take these situations as fusion states because their distance is zero in theory, but the fusion state may not happen because the surfactant can change droplets' surface tension and make the interface more stable (Mazutis et al. 2009) as shown in Fig. 7. The droplets outlet patterns are different for different controlling conditions.

Furthermore, Eq. (14) also indicates that different values of $\lambda_{0} / X$ (or input signals) correspond to different

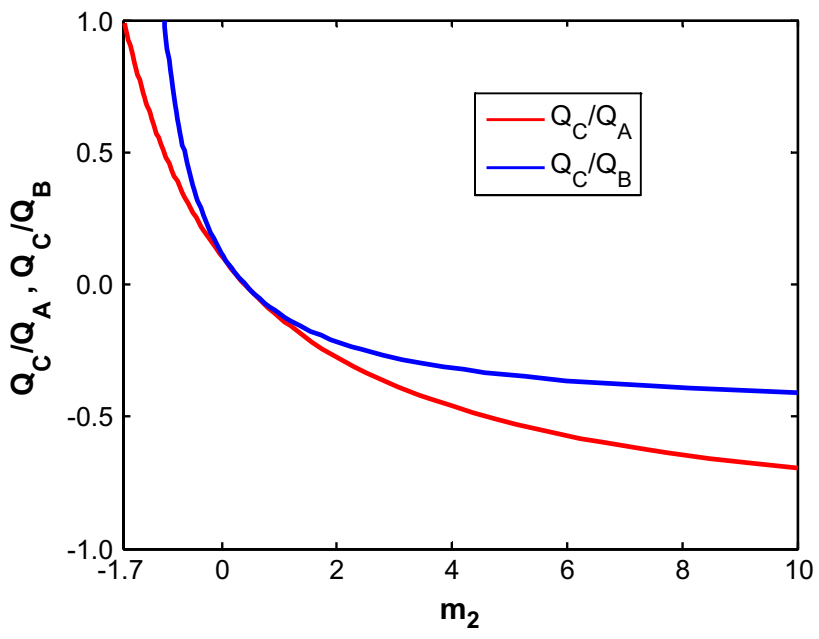

(a)

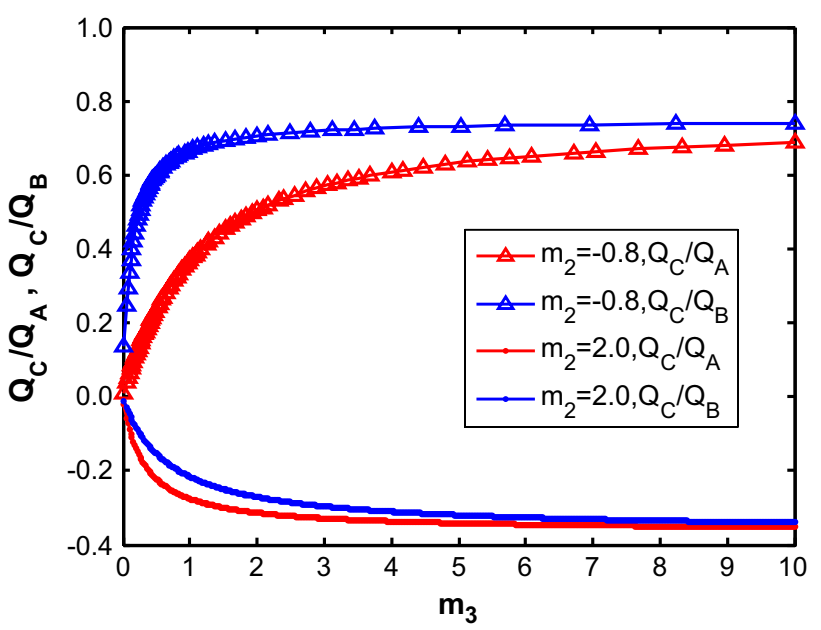

(b)

Fig. 9 Results of $Q_{C} / Q_{A}$ and $Q_{C} / Q_{B}$ which are the functions of $m_{2}$ and $m_{3}$, when there is no droplets going through branch $C$. a $Q_{C} / Q_{A}$ and $Q_{C} / Q_{B}$ are compared with 1 and -1 , respectively, to show the feasible range of $m_{2}$ under steady motion state of droplets and the experimental conditions. b The theory results about the influence of $L_{C}$ to the flow system under the experimental conditions. Here we assume $m_{4}=m_{3}$

$\lambda^{\mathrm{IV}}(n) / X$ and $\lambda^{\mathrm{IV}}(n+1) / X$ (or output signals) under the given conditions, that is, $\lambda^{\mathrm{IV}}(n) / X$ and $\lambda^{\mathrm{IV}}(n+1) / X$ are functions of $\lambda_{0} / X$. Figure 8 shows the input and output schematic results of droplets space intervals for $\lambda_{0} / X=0.052$ under the experimental conditions including $m_{2}=0.8$; Fig. $8 \mathrm{a}$ is input, while Fig. 8 b, c is output of analysis and experiment results respectively. A line segment (red or black) indicates the presence of a droplet. For $\lambda_{0} / X=0.052$, the droplet intervals of the new output droplet train are 0.0613 and 0.1046 , and they emerge in the staggered manner. 


\subsection{Motion state analysis of droplets}

Droplet will choose to enter the channel that has the instantaneous maximum flow rate when arriving at a junction. We assume that the droplet from either part does not go through branch $C$ in stage III, i.e., $Q_{C}<Q_{A}$ and $-Q_{C}<Q_{B}$. Thus we discuss the ratios of $Q_{C} / Q_{A}$ and $Q_{C} / Q_{B}$ and compare them with 1 and -1 , respectively; find the feasible range of $m_{2}$ under the conditions given before. We believe that if $Q_{C} / Q_{A}>1$ or $Q_{C} / Q_{B}<-1$, there will be droplets going through branch $C$, previous stability will be destroyed, and new stable states may be attained. An important reason that the model well describes the experimental data is that the fluctuations of the hydrodynamic resistances of the various arms of the loop remain small when compared to their mean values under the stability motion state. According to Eq. (10), we get Eq. (18) below:

$$
\left\{\begin{array}{l}
\frac{Q_{C}}{Q_{A}}=\frac{\left(\Gamma_{1} n_{A}+m_{3}\right) \frac{m_{1}+n_{1}}{m_{2}+n_{2}}-\left(\Gamma_{2} n_{B}+m_{4}\right)}{\left(\Gamma_{2} n_{B}+m_{4}+1\right) \frac{m_{1}+n_{1}}{m_{2}+n_{2}}+\left(\Gamma_{2} n_{B}+m_{4}\right)} ; \\
\frac{Q_{C}}{Q_{B}}=\frac{\left(\Gamma_{1} n_{A}+m_{3}\right) \frac{m_{1}+n_{1}}{m_{2}+n_{2}}-\left(\Gamma_{2} n_{B}+m_{4}\right)}{\left(\Gamma_{1} n_{A}+m_{3}+1\right)+\left(\Gamma_{1} n_{A}+m_{3}\right) \frac{m_{1}+n_{1}}{m_{2}+n_{2}}} .
\end{array}\right.
$$

where $n_{A}$ and $n_{B}$ are functions of $m_{2}$ and their solutions are in Appendix 2. We assume $L_{d 1}$ and $L_{d 2}$ are the additional excess resistive length of $R_{d 1}$ and $R_{d 2}$, respectively. Equation (18) can be changed to Eq. (19):

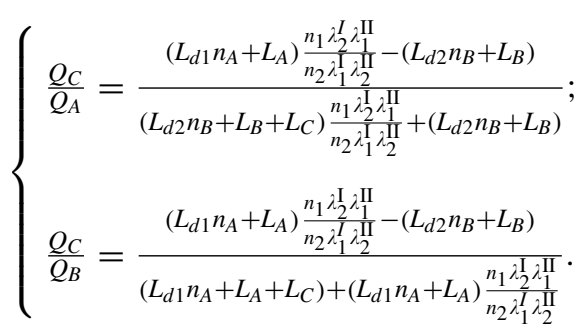

The conditions that $\lambda_{1}^{\mathrm{II}}$ and $\lambda_{2}^{I I}$ must satisfy in terms of $L_{A}$, $L_{B}, L_{C}, L_{d 1}$ and $L_{d 2}$ based on $Q_{C} / Q_{A}<1$ and $Q_{C} / Q_{B}>-1$ (no droplets flow through branch $C$ ) can be got:

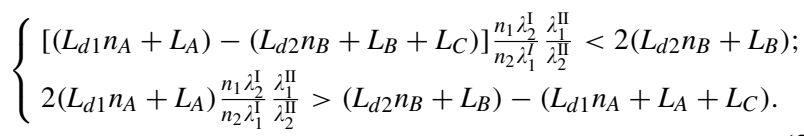

Equation (18) can be simplified based on the experimental conditions. Figure $9 \mathrm{a}$ shows the results of $Q_{C} / Q_{A}$ and $Q_{C} / Q_{B}$ that are functions of $m_{2}$. We find that $Q_{C} / Q_{A}=1$ in case $m_{2}=-1.7$ and it is the vertical asymptote of $Q_{C} / Q_{B}$; $Q_{C} / Q_{B}$ will not be less than -1 in the large range of $m_{2}$. The intersection point of $Q_{C} / Q_{A}$ and $Q_{C} / Q_{B}$ curves is (0.4, 0 ), i.e., the flow rate in branch $C$ is zero because of the symmetry of the structure and controlling conditions. Relative to $L_{A}\left(m_{3} L_{C}\right)$ and $L_{B}\left(m_{4} L_{C}\right)$, we also discuss the influence of $L_{C}$ to the flow system with the model. Here we assume

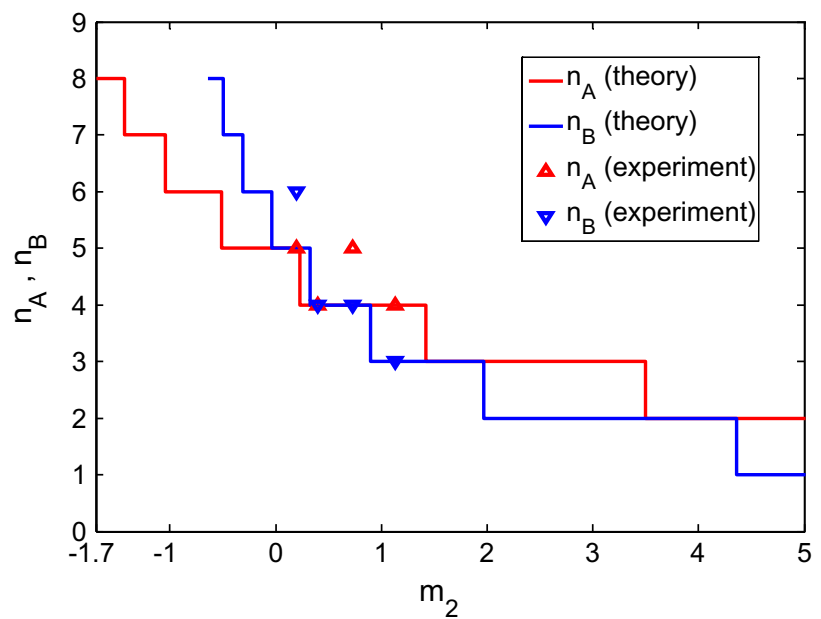

Fig. 10 Theory results about the average values of droplet number in branch $A\left(n_{A}\right)$ and branch $B\left(n_{B}\right)$ as functions of $m_{2}$. The experimental results are obtained by observation when $m_{2}$ equals to $0.2,0.4,0.7333$ and 1.1333. The result is under the experimental conditions

$m_{4}=m_{3}$ for convenient explanation and give the results that $Q_{C} / Q_{A}$ and $Q_{C} / Q_{B}$ are functions of $m_{3}$, as shown in Fig. $9 \mathrm{~b} ; m_{2}$ equals to -0.8 and 2.0 , respectively, under the experimental conditions. When $m_{2}$ equals to -0.8 , we have $Q_{C} / Q_{B}>Q_{C} / Q_{A}>0 ; Q_{C} / Q_{A}$ and $Q_{C} / Q_{B}$ increase with $m_{3}$ and tend to flat gradually. It indicates that the smaller $L_{C}$ becomes (relative to $L_{A}$ and $L_{B}$ ), the more likely the droplet train 1 has droplets go through branch $C$ than droplet train 2. It is because the flow rate of droplet train 1 is larger than that of droplet train 2, so there is flow rate through branch $C$ from node $c$ to node $d$ and increases relative to $Q_{A}$ when $L_{C}$ decreases, and droplets from train 1 have the trend go through branch $C$. When $m_{2}$ equals to 2.0, we have $Q_{C} / Q_{A}<Q_{C} / Q_{B}<0 ; Q_{C} / Q_{A}$ and $Q_{C} / Q_{B}$ decrease with $m_{3}$ and tend to flat gradually. It indicates that the smaller $L_{C}$ becomes (relative to $L_{A}$ and $L_{B}$ ), the more likely the droplet train 2 has droplets go through branch $C$ than droplet train 1 . We can also explain the reason as the case $m_{2}$ equals to -0.8 . When $m_{3}$ tends to zero, $c, d$ and $e$ become the same node (see Fig. 2), and Eq. (11) becomes Eq. (5). Figure 10 gives the results of $n_{A}$ and $n_{B}$ when $m_{2}$ ranges from -1.7 to 8 , the $n_{A}$ and $n_{B}$ curves also intersect when $m_{2}=0.4$. Meanwhile, the experimental results are also shown in Fig. 10 for the cases that $m_{2}$ equals to $0.2,0.4,0.7333$ and 1.1333. When $m_{2}$ varies in the range between -1.7 and 0.4 , Fig. 9a illustrates that $Q_{C} / Q_{B}>Q_{C} / Q_{A}>0$, i.e., $Q_{B}<Q_{A}$; we know $n_{A}<n_{B}$ from Appendix 2 because there is no droplets in branch $C, n_{A}$ curve is under $n_{B}$ curve. When $m_{2}>0.4, Q_{C} / Q_{A}<Q_{C} / Q_{B}<0$, so $Q_{A}<Q_{B}$ and $n_{A}>n_{B}, n_{A}$ curve is above $n_{B}$ curve. For the condition that $m_{3}$ does not equal to $m_{4}$, we can also get the results we want. 


\section{Conclusion}

In this paper, we studied droplets encoding and encounter (fusion in theory) problems in the microfluidic network. Two droplet trains are generated by the two same T-junctions, are controlled by two tuning flow rates and go through a looplike structure. Finally, a new droplet train is assembled in the outlet channel. We did mathematical analysis to the droplets transport process and set up a theoretical research model. This model reveals the relationship between the new droplet train's arrangement information (including droplets space intervals and droplet generation order) and the parameters, such as the tuning flow rates and so on. By doing the experimental research, we find the theoretical and experimental results agree well with each other, which verifies the validity of the theoretical model. The results show that droplets which may be generated at different moments can form encoding information in a certain tuning flow range, including the encounter one. After encounter, active or passive methods like electrocoalescence can be concerned, which can avoid many deficiencies of droplets fusion (Niu et al. 2009; Bremond et al. 2008; Niu et al. 2008; Zagnoni et al. 2009; Singh and Aubry 2007; Wang et al. 2009; Chen et al. 2013). Anyway, we make the research of droplet encoding and fusion together into one model. The range of the tuning flow rates and the influence of the loop-like structure size can be found by the model.

This theoretical model can provide guidance for the design of related microfluidic devices and research of droplet complex traffic problems; moreover, the new droplet train, containing specific information, can be used as the input signal of next stage to offer further multilevel studies. In addition, the work can be used to research cells, microorganisms, drug generation or chemical reactions, e.g., the interaction of normal cells and diseased cells after they are wrapped in droplets.

Acknowledgments The authors would like to acknowledge the support from the Hunan Provincial Natural Science Foundation for Young Scholars (No. 2015JJ3121), the Scientific Research Foundation of Xiangtan University (Nos. 13KZIKZ08022 and 13KZIKZ08029) and the National Natural Science Foundation of China (No. 11272321) for this work.

\section{Appendix 1: Droplet intervals in the dilution or concentration modules}

Figure 11 below is a simple network, and it is also a dilution or concentration module as Sessoms et al. (2009). One droplet train moves from channel 1 whose cross section is $S_{1}$ to channel 2 whose cross section is $S_{2}$, the slip factors of droplets equal to $\beta_{1}$ and $\beta_{2}$, respectively. The flow rate of channel 1 is $Q_{c 1}$, and the flow rate of channel 2 is

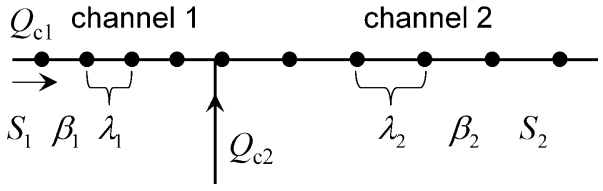

Fig. 11 A schematic diagram of droplet train dilution or concentration module

$Q_{c 1}+Q_{c 2}$ after dilution. $Q_{c 2}$ can be minus, but all the droplets from channel 1 will go through channel 2.

For the module, we assume that droplets entering frequency is $f_{1}$ while droplets exit frequency is $f_{2}$. The velocities of channel 1 and 2 are calculated as below:

$\left\{\begin{array}{l}V_{1}=\beta_{1} Q_{c 1} / S_{1} \\ V_{2}=\beta_{2}\left(Q_{c 1}+Q_{c 2}\right) / S_{2}\end{array}\right.$.

After $\Delta t$ time, the entering droplet number is $f_{1} \Delta t$, and the exit droplet number is $f_{2} \Delta t$. Meanwhile, the distance of any droplet from channel 1 and 2 is:

$\left\{L_{1}=V_{1} \Delta t=\beta_{1} Q_{c 1} \Delta t / S_{1} ;\right.$

$\left\{\begin{array}{l}L_{2}=V_{2} \Delta t=\beta_{2}\left(Q_{c 1}+Q_{c 2}\right) \Delta t / S_{2} .\end{array}\right.$

If we let $\lambda_{1}$ and $\lambda_{2}$ represent the space intervals between droplets which traffic in channel 1 and 2, respectively, then we have:

$\left\{\begin{array}{l}\lambda_{1}=L_{1} /\left(f_{1} \Delta t\right)=\beta_{1} Q_{c 1} /\left(S_{1} f_{1}\right) ; \\ \lambda_{2}=L_{2} /\left(f_{2} \Delta t\right)=\beta_{2}\left(Q_{c 1}+Q_{c 2}\right) /\left(S_{2} f_{2}\right) .\end{array}\right.$

We can get $f_{1}$ and $f_{2}$ from Eq. (23):

$\left\{f_{1}=\beta_{1} Q_{c 1} /\left(S_{1} \lambda_{1}\right)\right.$

$\left\{f_{2}=\beta_{2}\left(Q_{c 1}+Q_{c 2}\right) /\left(S_{2} \lambda_{2}\right)\right.$.

When droplets traffic in a microfluidic network, all the droplets number in the network will be stable under a stable traffic state. That is to say: $f_{1}=f_{2}$. Combining with Eq. (24), we have:

$\lambda_{2}=S_{1} \beta_{2}\left(Q_{c 1}+Q_{c 2}\right) \lambda_{1} /\left(S_{2} \beta_{1} Q_{c 1}\right)$

If the microchannels' cross sections are equal to $S$ and the slip factors of droplets in the microchannels are equal to $\beta$ (Bithi and Vanapalli 2010; Jeanneret et al. 2012), we have:

$\lambda_{2}=\left(Q_{c 1}+Q_{c 2}\right) \lambda_{1} / Q_{c 1}$

\section{Appendix 2: Droplet number in branch $A$ and branch $B$}

The total hydrodynamic resistances for branches $A, B$ and $C$ are $\left(n_{C}=0\right)$ :

$\left\{\begin{array}{l}R_{A}=\overline{R_{A}}+n_{A} R_{d 1} ; \\ R_{B}=\overline{R_{B}}+n_{B} R_{d 2} ; \\ R_{C}=\overline{R_{C}} .\end{array}\right.$ 
We also know that:

$\left\{\begin{array}{l}n_{A}=L_{A} / \lambda_{1}^{\text {III }} \\ n_{B}=L_{B} / \lambda_{2}^{\text {III }}\end{array}\right.$

Combining with Eqs. (8), (10) and (27), we have:

$\left\{\begin{array}{l}\lambda_{1}^{\mathrm{III}}=c_{1}\left(c_{3}+c_{4} R_{d 2} n_{B}\right) /\left(c_{5}+R_{d 1} n_{A}+R_{d 2} n_{B}\right) ; \\ \lambda_{2}^{\mathrm{III}}=c_{2}\left(c_{6}+c_{4} R_{d 1} n_{A}\right) /\left(c_{5}+R_{d 1} n_{A}+R_{d 2} n_{B}\right) .\end{array}\right.$

where the coefficients above are as follows:

$$
\left\{\begin{array}{l}
c_{1}=\lambda_{1}^{\mathrm{I}} / Q_{1} \\
c_{2}=\lambda_{2}^{\mathrm{I}} / Q_{2} \\
c_{3}=\left(Q_{1}+Q_{1 T}\right)\left(\overline{R_{B}}+\overline{R_{C}}\right)+\left(Q_{2}+Q_{2 T}\right) \overline{R_{B}} \\
c_{4}=Q_{1}+Q_{2}+Q_{1 T}+Q_{2 T} ; \\
c_{5}=\overline{R_{A}}+\overline{R_{B}}+\overline{R_{C}} ; \\
c_{6}=\left(Q_{1}+Q_{1 T}\right) \overline{R_{A}}+\left(Q_{2}+Q_{2 T}\right)\left(\overline{R_{A}}+\overline{R_{C}}\right) .
\end{array}\right.
$$

Substituting Eq. (29) into Eq. (28) yields

$\left\{\begin{array}{l}n_{A}=\left(c_{5}+R_{d 1} n_{A}+R_{d 2} n_{B}\right) L_{A} /\left[c_{1}\left(c_{3}+c_{4} R_{d 2} n_{B}\right)\right] \\ n_{B}=\left(c_{5}+R_{d 1} n_{A}+R_{d 2} n_{B}\right) L_{B} /\left[c_{2}\left(c_{6}+c_{4} R_{d 1} n_{A}\right)\right] .\end{array}\right.$

Finally, $n_{A}$ and $n_{B}$ can be got by solving Eq. (31).

\section{References}

Ahn K, Agresti J, Chong H, Marquez M, Weitz DA (2006) Electrocoalescence of drops synchronized by size-dependent flow in microfluidic channels. Appl Phys Lett 88:264105

Ajdari A (2004) Steady flows in networks of microfluidic channels: building on the analogy with electric circuits. C R Phys 5:539-546

Anna SL, Bontoux N, Stone HA (2003) Formation of dispersions using "flow focusing" in microchannels. Appl Phys Lett 82:364-366

Barbier V, Willaime H, Tabeling P (2006) Producing droplets in parallel microfluidic systems. Phys Rev E 74:046306

Behzad MD, Seyed-Allaei H, Ejtehadi MR (2010) Simulation of droplet trains in microfluidic networks. Phys Rev E 82:037303

Belloul M, Engl W, Colin A, Panizza P, Ajdari A (2009) Competition between local collisions and collective hydrodynamic feedback controls traffic flows in microfluidic networks. Phys Rev Lett 102:194502

Bithi SS, Vanapalli SA (2010) Behavior of a train of droplets in a fluidic network with hydrodynamic traps. Biomicrofluidics 4:044110

Bogojevic D, Chamberlain MD, Barbulovic-Nad I, Wheeler AR (2012) A digital microfluidic method for multiplexed cell-based apoptosis assays. Lab Chip 12:627-634

Bremond N, Thiam AR, Bibette J (2008) Decompressing emulsion droplets favors coalescence. Phys Rev Lett 100:024501

Bretherton F (1961) The motion of long bubbles in tubes. J Fluid Mech 10(2):166-188

Brouzes E, Medkova M, Savenelli N, Marran D, Twardowski M, Hutchison JB, Rothberg JM, Link DR, Perrimon N, Samuels ML (2009) Droplet microfluidic technology for single-cell highthroughput screening. PNAS 106:14195-14200
Chen G, Tan P, Chen S, Huang J, Wen W, Xu L (2013) Coalescence of pickering emulsion droplets induced by an electric field. Phys Rev Lett 110:064502

Cristobal G, Benoit J-P, Joanicot M, Ajdari A (2006) Microfluidic bypass for efficient passive regulation of droplet traffic at a junction. Appl Phys Lett 89:034104

Cybulski O, Garstecki P (2010) Dynamic memory in a microfluidic system of droplets traveling through a simple network of microchannels. Lab Chip 10:484-493

Engl W, Roche M, Colin A, Panizza P, Ajdari A (2005) Droplet traffic at a simple junction at low capillary numbers. Phys Rev Lett 95:208304

Fu T, Ma Y, Li HZ (2014) Hydrodynamic feedback on bubble breakup at a T-junction within an asymmetric loop. AIChE J 60(5):1920-1929

Fuerstman MJ, Garstecki P, Whitesides GM (2007) Coding/decoding and reversibility of droplet trains in microfluidic networks. Science 315:828-832

Garstecki P (2010) Microfluidics based microsystems: fundamentals and applications. Springer, Netherlands

Garstecki P, Fuerstman MJ, Whitesides GM (2005) Oscillations with uniquely long periods in a microfluidic bubble generator. Nat Phys 1:168-171

Glawder T, Elbuken C, Ren C (2011) Passive droplet trafficking at microfluidic junctions under geometric and flow asymmetries. Lab Chip 11:3774-3784

Gleichmann N, Malsch D, Horbert P, Henkel T (2014) Toward microfluidic design automation: a new system simulation toolkit for the in silico evaluation of droplet-based lab-on-a-chip systems. Microfluid Nanofluid 18:1095-1105

Hashimoto M, Whitesides GM (2010) Formation of bubbles in a multisection flow-focusing junction. Small 6:1051-1059

Huebner A, Srisa-Art M, Holt D, Abell C, Hollfelder F, de Mello AJ, Edel JB (2007) Quantitative detection of protein expression in single cells using droplet microfluidics. Chem Commun 12:1218-1220

Jeanneret R, Vest J-P, Bartolo D (2012) Hamiltonian traffic dynamics in microfluidic-loop networks. Phys Rev Lett 108:034501

Jousse F, Farr R, Link DR, Fuerstman MJ, Garstecki P (2006) Bifurcation of droplet flows within capillaries. Phys Rev E 74:036311

Labrot V, Schindler M, Guillot P, Colin A, Joanicot M (2009) Extracting the hydrodynamic resistance of droplets from their behavior in microchannel networks. Biomicrofluidics 3(1):257-265

Link DR, Anna SL, Weitz DA, Stone HA (2004) Geometrically mediated breakup of drops in microfluidic devices. Phys Rev Lett 92:054503

Link DR, Grasland-Mongrain E, Duri A, Sarrazin F, Cheng Z, Cristobal G, Marquez M, Weitz DA (2006) Electric control of droplets in microfluidic devices. Angew Chem 45(16):2556-2560

Liu K, Deng Y, Zhang N, Li S, Ding H, Guo F, Liu W, Guo S, Zhao $\mathrm{X}-\mathrm{Z}$ (2012) Generation of disk-like beads for cell encapsulation and manipulation using a droplet-based microfluidic device. Microfluid Nanofluid 13:761-767

Maddala J, Wang WS, Vanapalli SA, Rengaswamy R (2013) Traffic of pairs of drops in microfluidic ladder networks with fore-aft structural asymmetry. Microfluid Nanofluid 14:337-344

Mazutis L, Griffiths AD (2009) Preparation of monodisperse emulsions by hydrodynamic size fractionation. Appl Phys Lett 95:204103

Mazutis L, Baret J-C, Griffiths AD (2009) A fast and efficient microfluidic system for highly selective one-to-one droplet fusion. Lab Chip 9:2665-2672

Miller OJ, El Harrak A, Mangeat T, Baret J-C, Frenz L, El Debs B, Mayot E, Samuels ML, Rooney EK, Dieu P, Galvan M, Link DR, Griffiths AD (2012) High-resolution dose-response screening using droplet-based microfluidics. PNAS 109:378-383 
Mostowfi F, Khristov K, Czarnecki J, Masliyah J, Bhattacharjee S (2007) Electric field mediated breakdown of thin liquid films separating microscopic emulsion droplets. Appl Phys Lett 90:184102

Murran MA, Najjaran H (2012) Direct current pulse train actuation to enhance droplet control in digital microfluidics. Appl Phys Lett 101:144102

Niu X, Gulati S, Edel JB, deMello AJ (2008) Pillar-induced droplet merging in microfluidic circuits. Lab Chip 8:1837-1841

Niu X, Gielen F, deMello AJ, Edel JB (2009) Electro-coalescence of digitally controlled droplets. Anal Chem 81:7321-7325

Okushima S, Nisisako T, Torii T, Higuchi T (2004) Controlled production of monodisperse double emulsions by two-step droplet breakup in microfluidic devices. Langmuir 20(23):9905-9908

Park C-W, Homsy G (1984) Two-phase displacement in Hele Shaw cells: theory. J Fluid Mech 139(1):291-308

Prakash M, Gershenfeld N (2007) Microfluidic bubble logic. Science 315:832-835

Salkin L, Schmit A, Courbin L, Panizza P (2013) Passive breakups of isolated drops and one-dimensional assemblies of drops in microfluidic geometries: experiments and models. Lab Chip 13(15):3022-3032

Schindler M, Ajdari A (2008) Droplet traffic in microfluidic networks: a simple model for understanding and designing. Phys Rev Lett 100:044501

Sessoms DA, Belloul M, Engl W, Roche M, Courbin L, Panizza P (2009) Droplet motion in microfluidic networks: hydrodynamic interactions and pressure-drop measurements. Phys Rev E 80:016317

Sessoms DA, Amon A, Courbin L, Panizza P (2010) Complex dynamics of droplet traffic in a bifurcating microfluidic channel: periodicity, multistability, and selection rules. Phys Rev Lett 105:154501

Singh P, Aubry N (2007) Transport and deformation of droplets in a microdevice using dielectrophoresis. Electrophoresis 28:644-657

Smith BJ, Gaver DP (2010) Agent-based simulations of complex droplet pattern formation in a two-branch microfluidic network. Lab Chip 10:303-312
Song H, Tice JD, Ismagilov RF (2003) A microfluidic system for controlling reaction networks in time. Angew Chem 42:768-772

Song K, Zhang L, Hu G (2012) Modeling of droplet traffic in interconnected microfluidic ladder devices. Electrophoresis 33:411-418

Takinoue M, Takeuchi S (2011) Droplet microfluidics for the study of artificial cells. Anal Bioanal Chem 400:1705-1716

Thorsen T, Roberts RW, Arnold FH, Quake SR (2001) Dynamic pattern formation in a vesicle-generating microfluidic device. Phys Rev Lett 86:4163-4166

Wang W, Yang C, Li CM (2009) On-demand microfluidic droplet trapping and fusion for on-chip static droplet assays. Lab Chip 9:1504-1506

Willaime H, Barbier V, Kloul L, Maine S, Tabeling P (2006) Arnold tongues in a microfluidic drop emitter. Phys Rev Lett 96:054501

Wong H, Radke C, Morris S (1995) The motion of long bubbles in polygonal capillaries. Part 1. Thin films. J Fluid Mech 292:71-94

Wu Y, Fu T, Zhu C, Ma Y, Li HZ (2012) Asymmetrical breakup of bubbles at a microfluidic T-junction divergence: feedback effect of bubble collision. Microfluid Nanofluid 13:723-733

Wu Y, Fu T, Zhu C, Ma Y, Li HZ (2014) Bubble coalescence at a microfluidic T-junction convergence: from colliding to squeezing. Microfluid Nanofluid 16(1-2):275-286

Yamada M, Doi S, Maenaka H, Yasuda M, Seki M (2008) Hydrodynamic control of droplet division in bifurcating microchannel and its application to particle synthesis. J Colloid Interface Sci 321:401-407

Zagnoni M, Baroud CN, Cooper JM (2009) Electrically initiated upstream coalescence cascade of droplets in a microfluidic flow. Phys Rev E 80:046303

Zanella A, Biral A (2014) Design and analysis of a microfluidic bus network with bypass channels. IEEE International Conference on Communications 3993-3998

Zheng B, Tice JD, Ismagilov RF (2004) Formation of droplets of alternating composition in microfluidic channels and applications to indexing of concentrations in droplet-based assays. Anal Chem 76:4977-4982

Zhou H, Yao S (2013) Electrostatic charging and control of droplets in microfluidic devices. Lab Chip 13:962-969 